\title{
Interés vocacional-profesional y rendimiento académico en los estudiantes de la Universidad Nacional del Centro del Perú, 2012
}

\author{
Professional vocational interest and academic performance in the \\ students from the Universidad Nacional del Centro del Perú, 2012
}

Félix Rodríguez Isidro

Universidad Nacional del Centro del Perú

\section{RESUMEN}

Objetivo: Determinar la relación que existe entre el interés vocacional-profesional y el rendimiento académico en los cursos de especialidad de los estudiantes del primer y tercer ciclo de la Escuela Profesional de Educación Primaria de la Facultad de Educación de la Universidad Nacional del Centro del Perú - Huancayo 2012. Método: La investigación fue de nivel descriptivo correlacional. La muestra estuvo constituida por los estudiantes del primer y tercer ciclo de la Facultad de Educación de la UNCP durante el semestre 2012. La selección se realizó mediante muestreo aleatorio simple. Resultados: Con un nivel de significancia del $5 \%$, hay evidencia estadística suficiente para afirmar que existe correlación positiva entre el interés vocacional-profesional y el rendimiento académico en los cursos de especialidad de los estudiantes del primer y tercer ciclo de la Facultad de Educación de la Universidad Nacional del Centro del Perú. Conclusiones: El interés vocacionalprofesional influye favorable y significativamente en el rendimiento académico en los cursos de especialidad que desarrollan los estudiantes del primer y tercer ciclo de la Facultad de Educación de la UNCP, semestre 2012. El rendimiento académico en los cursos de especialidad es de normal promedio (media de 13,43) en los estudiantes del primer y tercer ciclo de la Facultad de Educación de la UNCP - Huancayo. 


\section{ABSTRACT}

Objective: To determine the relationship between vocational-professional interest and the academic performance in specialism subjects of first and third cycle students from the Primary Education Professional School in the Education Faculty at the "Universidad Nacional del Centro del Perú" - Huancayo 2012. Methods: The research has correlational descriptive level. The sample was formed by first and third cycle students from the Education Faculty at UNCP during the semester 2012. The selection was made by simple random sampling. Results: With a significance level of $5 \%$, there is enough statistical evidence to affirm that there is positive correlation between the vocationalprofessional interest and the academic performance in specialism subjects of the first and third cycle students from the Education Faculty at the "Universidad Nacional del Centro del Perú". Conclusions: The vocational-professional interest affects positive and significantly in the academic performance in specialism subjects that have the first and third year students from the Education Faculty at the UNCP, semester 2012. The academic performance in specialism subjects is in normal average (mean of 13,43) Table No. 5 and 6 in first and third year students from the Education Faculty at the UNCP - Huancayo.

Keywords: Vocational Interest, academic performance, university students.

\section{INTRODUCCIÓN}

En muchas instituciones de nivel universitario de la ciudad de Huancayo se observa que en los últimos años se ha implementado diferentes tipos y niveles de selección de los estudiantes para su ingreso a una especialidad en la Facultad de Educación; sin embargo, no existe un estudio sistemático y actualizado sobre la naturaleza y características de estos exámenes de selección o pruebas de admisión que involucre los intereses vocacionales y profesionales del candidato. De la misma forma, se observa que muchos estudiantes ingresan a una especialidad solo como un medio de "efecto trampolín" para pasarse a otras especialidades, 0 en otros casos, ingresan a una especialidad por simple sugerencia o imposición de sus padres sin tener interés vocacional-profesional adecuado para dicha especialidad.

Estas dificultades y contradicciones repercuten negativamente en el proceso de aprendizaje y rendimiento académico de los estudiantes en los diversos cursos de especialidad, caso sobre el que tampoco se ha hallado investigaciones ni estudios que reflejen esta realidad y así plantear las alternativas más adecuadas.

En la Facultad de Educación de la UNCP de Huancayo también se viene aplicando exámenes de selección para el ingreso de los estudiantes en las diversas especialidades, sin explorar el interés vocacionalprofesional, y en muchas ocasiones ellos se ubican para completar las vacantes. Como consecuencia de esta situación, se observa un bajo nivel de rendimiento académico en los estudiantes, en algunos casos se ha encontrado deserciones, traslados y descontento.

Por esta razón la conveniencia de investigar en forma descriptiva y analítica el interés vocacional-profesional y el rendimiento académico de los estudiantes en la Escuela Profesional de Educación Primaria de la Facultad de Educación de la UNCP, con el propósito de conocer esta realidad y plantear las alternativas convenientes.

La psicología como marco de reflexión científica sobre la conducta vocacional ha situado los intereses en un lugar privilegiado, otorgándoles un valor nuclear en la decisión vocacional del individuo, caso que resulta fundamental para el análisis de los procesos de elección vocacional y especialmente en los denominados momentos vocacionales críticos. Son en estos momentos de "encrucijada" cuando el estudiante cambia de etapa y tiene 
que elegir itinerarios educativos con distinto componente profesionalizador, donde deberá decidirse entre la amplia lista de titulaciones universitarias y la formación profesional específica de grado universitario.

Al revisar la investigación sobre los factores mediacionales de los intereses profesionales, encontramos una tendencia a realizar meros estudios correlacionales, que se centran aisladamente en los componentes que afectan a su determinación; así disponemos de estudios sobre la relación unidireccional entre variables como aptitudes, género, estatus socio familiar, rendimiento académico, personalidad, estilos cognitivos, autoeficacia, autoestima y otras que pueden afectar directamente a los intereses profesionales. Sin embargo, se han realizado muy pocas investigaciones sobre modelos que integren las interacciones e interrelaciones entre tales componentes.

A nivel nacional podemos citar a Damaris Díaz, y su tesis titulada: "Perfil vocacional y rendimiento escolar en universitarios en la universidad michoacana de San Nicolás de Hidalgo, para optar el grado académico de Magíster en Psicología Educativa. En su conclusión $\mathrm{N}^{\circ} 2$ define que los intereses vocacionales señalan dos tendencias: por un lado, muestra que el rendimiento escolar en la carrera de Psicología es mayor cuando los alumnos mantienen un menor interés en las áreas de mecánico y cálculo y, por otro, los alumnos que obtienen un mayor rendimiento reflejan mayor interés en las áreas persuasivo, musical y servicio social.

Nos encontramos, por tanto, con una necesidad no suficientemente cubierta aún por la teoría de la orientación profesional, la necesidad de desarrollar modelos teóricos de los mediadores más significativos en la configuración de los intereses profesionales, que adopten una perspectiva más global, más allá del simple listado de taxonomías de componentes importantes, para ofrecernos una información explicativa de cómo operan los componentes de manera sistemática. Es decir, se nos presenta el problema de integrar dentro de un modelo conceptual de correlación de las diversas variables que actúan como mediadores en la configuración de los intereses profesionales de los estudiantes y proceder a su contrastación.

Establecer un modelo de correlaciones de las dos variables que intervienen como mediadores de los intereses profesionales, exige, epistemológicamente, en primer lugar, decidir el marco teórico desde el que debemos fundamentar el modelo postulado, para no caer en una mera acumulación de datos empíricos sin relevancia científica.

Establecer un modelo de correlaciones de las dos variables que intervienen como mediadores de los intereses profesionales, exige, epistemológicamente, en primer lugar, decidir el marco teórico desde el cual fundamentar el modelo postulado, para no caer en una mera acumulación de datos empíricos sin relevancia científica. Se ha optado como marco de referencia de la investigación la teoría cognitiva social formulada por Bandura (1) por un lado, y la teoría del autoconcepto vocacional de Super (2) por otro, y partiendo de éstas, nos basaremos en la concepción del Interés vocacional - profesional de Vicuña (3), quien asume la vocación como un constructo teórico cuyo sustento es el interés, entendido como un componente adquirido de la motivación que actúa cuando se toma conciencia de su ausencia - distanciamiento, movilizando los mecanismos de la impulsión haciendo que la persona dirija su comportamiento para conseguirlo. El interés puede ser intrínseco o extrínseco.

Los intereses profesionales, más allá de las predisposiciones genéticas, son fundamentalmente aprendizajes sociales, en el marco sociohistórico de una determinada cultura. Como pretendemos comprobar, los factores socioambientales de género, estatus sociofamiliar, estatus académico y el centro donde se realizan los estudios, por su relación directa con los intereses profesionales, pueden afectar significativamente a las diferencias que encontramos en la de los mismos durante 
la etapa de educación secundaria. Ahora bien, es necesario también comprender las variables de proceso, cognitivomediacionales, que en el marco de un conjunto de factores socioambientales, operan en los estudiantes a la hora de cristalizar en una determinada disposición sus intereses profesionales.

El conocimiento de estos procesos cognitivomediacionales, es decir la relación de su rendimiento académico como muestra de su madurez cognoscitiva, nos permitirá desde el ámbito universitario diseñar programas educativos en el marco de la "educación para la carrera" (insertos en el propio configuran la estructura de los intereses profesionales de los estudiantes, puede permitirnos además la elaboración de programas de educación familiar que faciliten la superación de prácticas y estereotipos sociofamiliares que sesgan la distribución normal de los intereses profesionales de nuestros adolescentes y jóvenes. Asimismo, aunque resulte ambicioso, no por ello es menos necesario, la fundamentación de las propuestas - sugerencias nos permitiría proponer recomendaciones para que desde los medios de comunicación, principalmente desde la publicidad y las series televisivas, se modifiquen determinados estereotipos

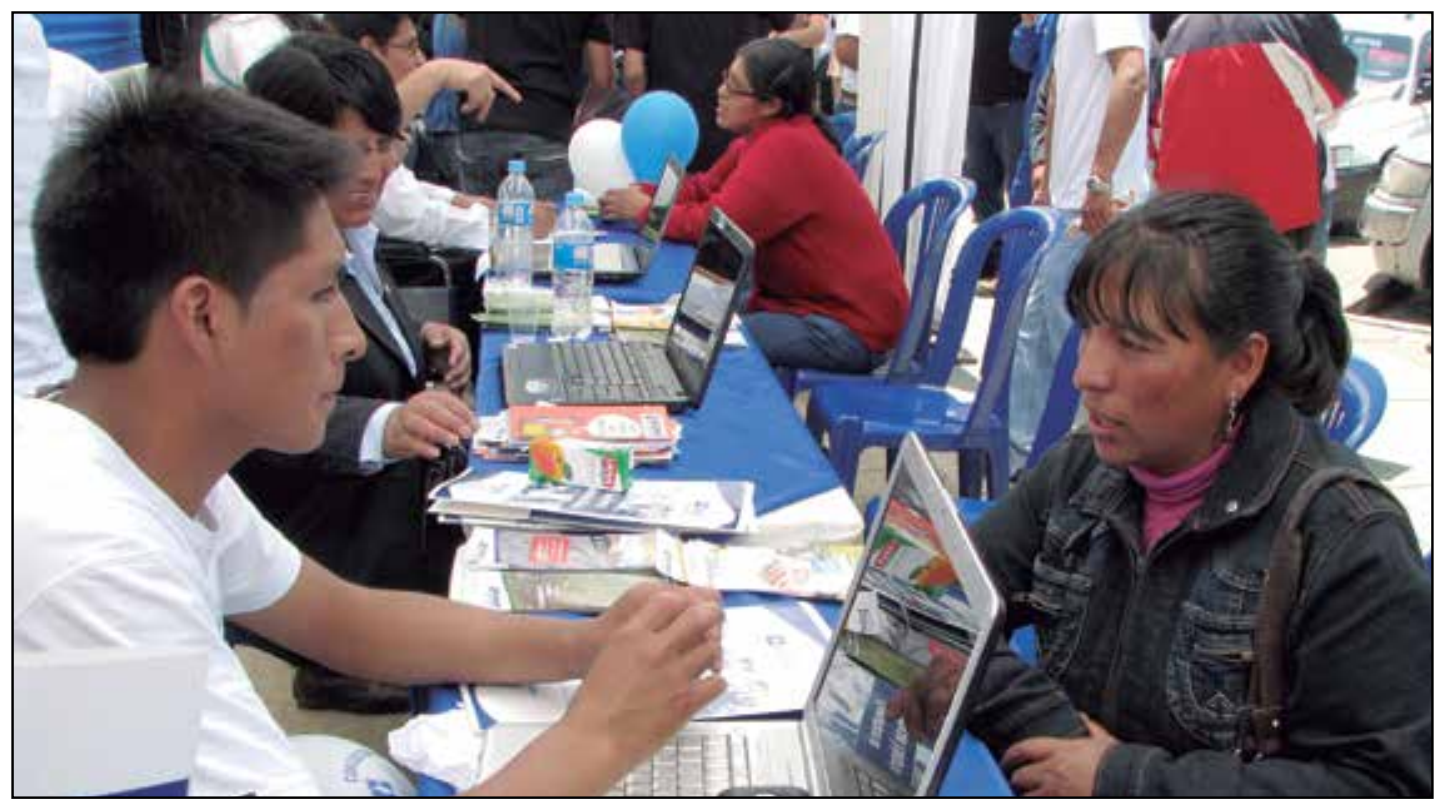

Figura $\mathrm{N}^{\circ}$ 1: Las campañas de orientación vocacional promueven el interés vocacional, variable que influye significativamente en el rendimiento de los universitarios.

currículo), que hagan a los estudiantes más conscientes (y por ende más libres) de cómo han llegado a construir sus cogniciones y afectos sobre las ofertas de actividades profesionales que la sociedad les ofrece. Conciencia necesaria pero no suficiente, como primer paso, para aprovechar las oportunidades profesionales de un mercado laboral flexible y cambiante como el que va a caracterizar sus vidas profesionales en el marco de la nueva sociedad global.

Una mejor comprensión de los procesos de aprovechamiento académico que socioprofesionales, que constituyen experiencias vicarias que a través de los procesos del modelado social ejercen un significativo influjo en los futuros intereses profesionales de nuestros jóvenes.

\section{MATERIAL Y MÉTODOS}

El tipo de este estudio es descriptivo correlacional, debido a que busca una 
relación entre el interés vocacionalprofesional y el rendimiento académico de los cursos de especialidad que desarrollan los estudiantes del primer y tercer ciclo de la Facultad de Educación de la UNCP Huancayo.

El diseño de la investigación es no experimental porque no existe una variable a la cual se va a manipular; es decir, se observan situaciones ya existentes, no provocadas como en los estudios experimentales $\circ$ cuasiexperimentales. En la investigación no experimental las variables independientes ocurren y no es posible manipularlas ni tener control sobre ellas, porque ya sucedieron al igual que sus efectos. Es de corte transversal porque se recoge la información en un solo momento en el tiempo con el propósito de describir las variables y analizar su incidencia e interrelación en un momento dado.

Para la contrastación de las hipótesis se usó técnicas estadísticas donde la mayoría de estos análisis estuvieron centrados en los usos y la interpretación de los métodos, más que en los procedimientos de cálculo, debido a que en la actualidad los análisis se realizan con la ayuda de una computadora y no manualmente.

La población estuvo constituida por los estudiantes del primer y tercer ciclo de la Escuela Profesional de Educación Primaria de Facultad de Educación de la UNCP de la ciudad de Huancayo, quienes representan un total de 51 .

La muestra estuvo representada por los estudiantes del primer y tercer ciclo de la Escuela Profesional de Educación Primaria de la Facultad de Educación de la UNCP de la ciudad de Huancayo, constituidos por un

Tabla $N^{\circ}$ 1: Distribución de la muestra de estudiantes por ciclo de estudio.

\begin{tabular}{lcc}
\hline Ciclo & $\begin{array}{c}\mathrm{N}^{\circ} \text { de } \\
\text { estudiantes }\end{array}$ & Porcentaje \\
\hline Ciclo I & 15 & $42,9 \%$ \\
Ciclo III & 20 & $57,1 \%$ \\
\hline Total & 35 & $100,0 \%$ \\
\hline
\end{tabular}

total de 35 (tabla $\left.N^{\circ} 1\right)$ y pertenecientes al semestre 2012-1.

El diseño muestral fue probabilístico estratificado, donde cada elemento de la población o cada estudiante en este caso tiene la misma probabilidad de ser seleccionado. La estratificación se realizó por ciclo de estudio, considerando que los estudiantes de un ciclo y otro no llevan las mismas asignaturas.

El tamaño de la muestra fue de 35 estudiantes. Esta cantidad se distribuyó mediante afijación proporcional; es decir, de los 35 estudiantes de Educación, 15 fueron seleccionados del primer ciclo y 20 del tercer ciclo.

La selección de la muestra se realizó mediante muestreo aleatorio simple, tomando como marco muestral el listado de estudiantes en cada ciclo y generando una lista de números aleatorios con el

Tabla $N^{\circ} 2$ : Distribución de la población y la muestra por cada ciclo.

\begin{tabular}{cccc}
\hline \multicolumn{2}{c}{ Población } & \multicolumn{2}{c}{ Muestra } \\
\hline Estrato & Tamaño & Estrato & Tamaño \\
\hline N1 & 21 & $\mathrm{n} 1$ & 15 \\
N2 & 30 & $\mathrm{n} 2$ & 20 \\
\hline N & 51 & $\mathrm{n}$ & 35
\end{tabular}

Fuente: Nómina de matrícula oficial de la UNCP.

programa Excel, que permitió identificar qué estudiantes deberían ser seleccionados (tabla $\mathrm{N}^{\circ} 2$ ).

En la recolección de datos, se aplicó la técnica de Psicometría y su instrumento, el cuestionario de exploración vocacional, inventario de intereses vocacionales CASM adaptado y revisado en Huancayo el 2010,

Tabla N ${ }^{\circ}$ 3: Distribución de la muestra de estudiantes por ciclo de estudio.

\begin{tabular}{lcccc}
\hline Género & Ciclo I & Ciclo III & Total & Porcentaje \\
\hline masculino & 1 & 4 & 5 & $14 \%$ \\
Femenino & 14 & 16 & 30 & $86 \%$ \\
\hline Total & 15 & 20 & 35 & $100,0 \%$ \\
\hline
\end{tabular}


para estudiantes del primer ciclo de la Facultad de Educación. Asimismo, se aplicó la técnica de análisis documentario, cuyo instrumento es el registro de notas.

Tabla N²:Distribución porcentual de los estudiantes según su interés vocacional por las Ciencias Sociales en cada ciclo y en total.

\begin{tabular}{|c|c|c|c|c|}
\hline \multirow{2}{*}{$\begin{array}{l}\text { Ciclo de } \\
\text { estudios }\end{array}$} & \multicolumn{3}{|c|}{ Interés por las CC.SS } & \multirow{2}{*}{ Total } \\
\hline & Alto & Indeciso & Bajo & \\
\hline Ciclo I & $47 \%$ & $40 \%$ & $13 \%$ & $100 \%$ \\
\hline Ciclo III & $30 \%$ & $45 \%$ & $25 \%$ & $100 \%$ \\
\hline Total & $77 \%$ & $43 \%$ & $20 \%$ & $100 \%$ \\
\hline
\end{tabular}

\section{RESULTADOS}

\section{Presentación y análisis de las características sociodemográficas}

En la Tabla $\mathrm{N}^{\circ} 2$ puede observarse que el número de estudiantes en el tercer ciclo es mayor que en el primero, lo que indica que el número de ingresantes a la carrera de Educación en la UNCP está disminuyendo y la razón puede ser por la falta de motivación e interés por la docencia.

Otro aspecto interesante es el número de estudiantes varones en educación primaria. Según la Tabla $N^{\circ} 3$ los varones representan apenas el $14 \%$ de la población estudiantil, mientras que la presencia de las mujeres en esta especialidad alcanza el $86 \%$.

\section{Presentación y análisis de la variable interés vocacional- profesional por las CC.SS.}

La tabla $\mathrm{N}^{\circ} 4$ muestra los resultados de la aplicación del Inventario de Intereses Vocacionales CASM, muestran que el $77 \%$ de los estudiantes en ambos ciclos tiene un alto interés por las CC.SS., área en el que se encuentra ubicado la carrera profesional.

Tabla $N^{\circ}$ 5: Estadísticas de las notas obtenidas en los cursos de especialidad por los estudiantes del primer ciclo en el semestre 2012-1.

\begin{tabular}{|c|c|c|c|c|c|}
\hline Asignatura & Mín & Mín & Media & Desv. típ & Coef. var. \\
\hline Pedagogía y Ciencias de la Educación & 12,0 & 15,3 & 13,27 & 1,02 & $8 \%$ \\
\hline Corrientes pedagógicas y modelos didácticos & 11,0 & 15,7 & 13,19 & 1,21 & $9 \%$ \\
\hline Salud e higiene para educación primaria & 12,3 & 14,7 & 13,01 & 0,65 & $5 \%$ \\
\hline Aprestamiento para lectura, escritura y matemática & 11,0 & 13,7 & 12,16 & 0,83 & $7 \%$ \\
\hline Rendimiento académico & 12,2 & 14,3 & 12,91 & 0,56 & $4 \%$ \\
\hline
\end{tabular}

Fuente: Actas promocionales de la Universidad Nacional del Centro del Perú.

Tabla $N^{\circ}$ 6: Estadísticas de las notas obtenidas en los cursos de especialidad por los estudiantes del tercer ciclo en el semestre 2012-1.

\begin{tabular}{|c|c|c|c|c|c|}
\hline Asignatura & Mín & Mín & Media & Desv. típ & Coef. var. \\
\hline Diseño y diversificación curricular & 12,0 & 17,0 & 14,65 & 1,42 & $10 \%$ \\
\hline Psicología educativa y del aprendizaje & 15,3 & 16,7 & 15,96 & 0,30 & $2 \%$ \\
\hline Didáctica de la matemática & 9,3 & 16,3 & 13,18 & 1,70 & $13 \%$ \\
\hline Didáctica del lenguaje & 10,3 & 13,0 & 11,93 & 0,67 & $6 \%$ \\
\hline Rendimiento académico & 12,3 & 14,9 & 13,94 & 0,71 & $5 \%$ \\
\hline
\end{tabular}

Fuente: Actas promocionales de la Universidad Nacional del Centro del Perú. 


\section{Presentación y análisis de la variable rendimiento académico}

La Tabla $N^{\circ} 5$ muestra las estadísticas de las notas de los estudiantes del primer ciclo en los cursos de especialidad. Con una nota promedio de 12,91 y una desviación estándar de 0,56, el resultado puede considerarse como rendimiento normal promedio por parte de los estudiantes. El 13,33 \% de alumnos alcanzaron notas de 15 en las asignaturas de Pedagogía y Ciencias de la Educación y Corrientes pedagógicas y modelos didácticos. al cual pertenece la carrera profesional de Educación.

\section{DISCUSIÓN}

Se ha demostrado con un nivel de significancia del $5 \%$ que existe una correlación positiva entre el interés vocacional-profesional y rendimiento académico en los cursos de especialidad de los estudiantes de la Facultad de Educación de la UNCP - Huancayo 2012.

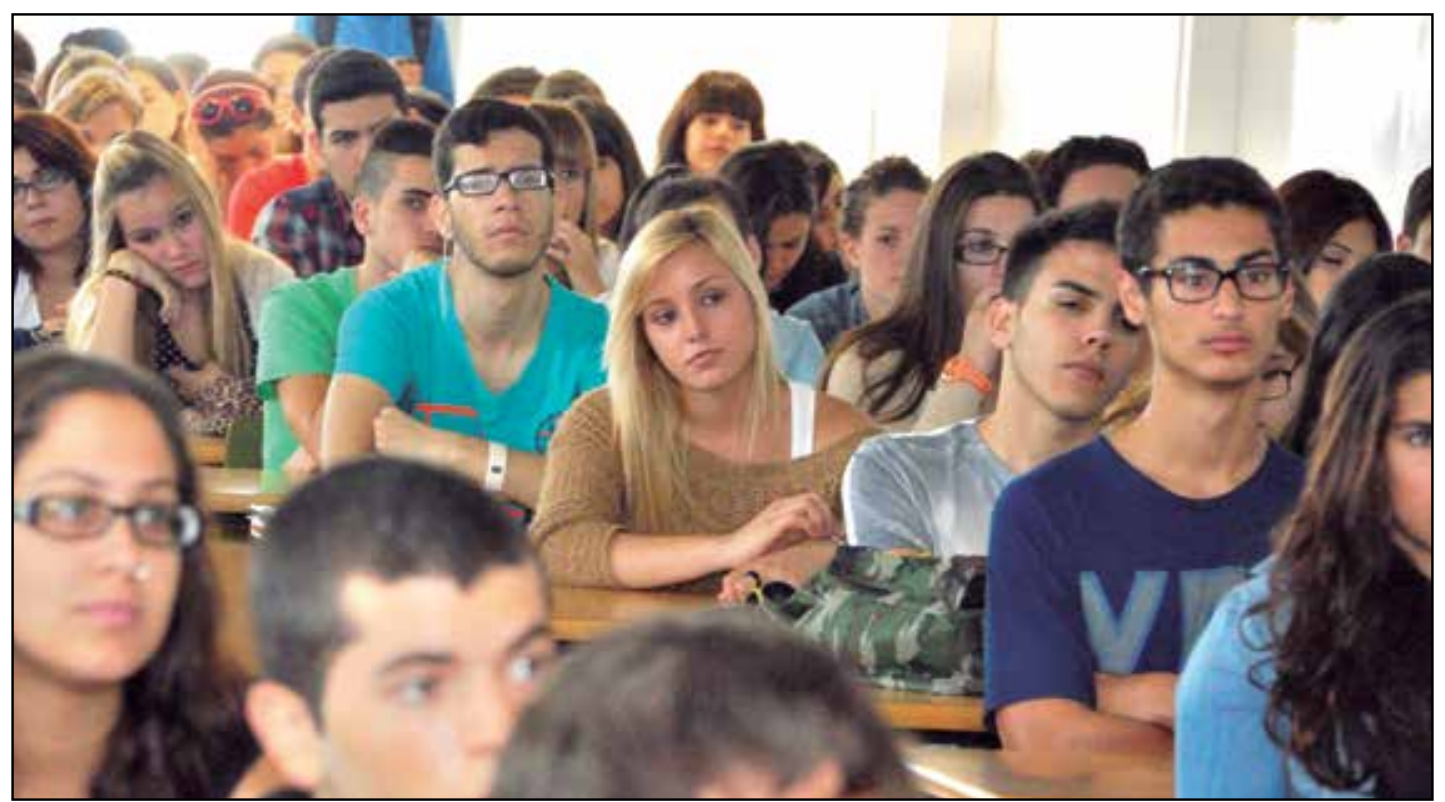

Figura $\mathrm{N}^{\circ}$ 2: Charla de orientación vocacional.

Fuente: Diario El País, España, http://blogs.elpais.com/ayuda-al-estudiante/2013/05/elegir-carrera-ya-no-es-lo-que-era.html

La Tabla № 6 muestra las estadísticas de las notas de los estudiantes del tercer ciclo en los cursos de especialidad. Con una nota promedio de 13,94 y una desviación estándar de 0,71 , las notas son superiores a la de los estudiantes del primer ciclo. El $10 \%$ de estudiantes alcanzaron notas de 16 y 17 en las asignaturas de Diseño y diversificación curricular y Psicología educativa y del aprendizaje, esto corrobora a que los estudiantes del tercer ciclo poseen en un $77 \%$ el interés vocacionalprofesional en el área de ciencias sociales
También se ha logrado comprobar que la preferencia vocacional de la persona es un proceso evolutivo que expresa la idea de la persona que es, ya que al ingresar en una profesión trata de poner en práctica el concepto que tiene de sí mismo (autoconcepto), y estableciéndose ya en los cursos de especialidad consigue la actualización de sus conocimientos que se ve expresado en su rendimiento académico. Estas preferencias, cambian con el tiempo y la experiencia, aunque los conceptos de sí mismos son bastante estables desde la adolescencia hasta madurez como lo 
manifiesta Super.

El interés vocacional-profesional influye favorable y significativamente en el rendimiento académico en los cursos de especialidad que desarrollan los estudiantes del primer y tercer ciclo de la Facultad de Educación de la UNCP - Huancayo 2012.

Los alumnos que tienen una adecuada orientación vocacional en la educación básica regular y en la admisión para los estudios universitarios obtienen mejor rendimiento académico en los cursos de especialidad ya que éstas son de gran motivación por desarrollar capacidades y competencias que pertenecen al área de su interés vocacional - profesional.

Ello demuestra que los adolescentes con una adecuada orientación en los intereses vocacionales y profesionales, pueden tomar decisiones con respecto a su futuro profesional ya que esto se hace en las edades de la adolescencia como lo dice Rocaber (4), corroborado por Gil y Hernández (5), quien conjetura que los intereses vocacionales y profesionales ya se encuentran cristalizadas al término de la secundaria.

\section{REFERENCIAS BIBLIOGRÁFICAS}

1. Bandura A. Pensamiento y acción, fundamentos sociales. Barcelona: Martínez Roca; 1986.

2. Super DE. A vocational interest patterns: A study in the psychology of avocations. Palo Alto CA: Stanford University Press. 1940.

3. Vicuña L. Elaboración del inventario de intereses vocacionales para carreras técnicas y de oficio. Revista de investigación en Psicología. 2002; 05(01): 117-140.

4. Rocabert, E. La opción universitaria: un sistema de exploración de la conducta vocacional basado en los intereses y preferencias vocacionales. [Tesis doctoral], Valencia: Valencia; 1987.

5. Gil JM. Información vocacional: estrategias y técnicas de intervención. In F. R. Martínez. Madrid: Síntesis; 1995. 